\title{
BMJ Open Barriers and facilitators to orthopaedic surgeons' uptake of decision aids for total knee arthroplasty: a qualitative study
}

\author{
Samantha Bunzli, ${ }^{1}$ Elizabeth Nelson, ${ }^{2}$ Anthony Scott, ${ }^{3}$ Simon French, ${ }^{4}$ \\ Peter Choong, ${ }^{1}$ Michelle Dowsey ${ }^{1}$
}

To cite: Bunzli S, Nelson E, Scott A, et al. Barriers and facilitators to orthopaedic surgeons' uptake of decision aids for total knee arthroplasty: a qualitative study. BMJ Open 2017;7:e018614. doi:10.1136/ bmjopen-2017-018614

- Prepublication history and additional material for this paper are available online. To view these files, please visit the journal online (http://dx.doi. org/10.1136/bmjopen-2017018614).

Received 10 July 2017 Revised 9 October 2017 Accepted 10 October 2017

\section{(a) CrossMark}

${ }^{1}$ Department of Surgery, The University of Melbourne, St Vincent's Hospital, Melbourne, Victoria, Australia

${ }^{2}$ Department of Orthopaedics, St Vincent's Hospital, Melbourne, Victoria, Australia

${ }^{3}$ Faculty of Business and Economics, Melbourne Institute of Applied Economic and Social Research, The University of Melbourne, Melbourne, Victoria, Australia

${ }^{4}$ Faculty of Health Sciences, School of Rehabilitation Therapy, Queen's University, Kingston, Ontario, Canada

Correspondence to Dr Samantha Bunzli; sbunzli@unimelb.edu.au

\section{ABSTRACT}

Objectives The demand for total knee arthroplasty (TKA) is increasing. Differentiating who will derive a clinically meaningful improvement from TKA from others is a key challenge for orthopaedic surgeons. Decision aids can help surgeons select appropriate candidates for surgery, but their uptake has been low. The aim of this study was to explore the barriers and facilitators to decision aid uptake among orthopaedic surgeons.

Design A qualitative study involving face-to-face interviews. Questions were constructed on the Theoretical Domains Framework to systematically explore barriers and facilitators.

Setting One tertiary hospital in Australia.

Participants Twenty orthopaedic surgeons performing TKA.

Outcome measures Beliefs underlying similar interview responses were identified and grouped together as themes describing relevant barriers and facilitators to uptake of decision aids.

Results While prioritising their clinical acumen, surgeons believed a decision aid could enhance communication and patient informed consent. Barriers identified included the perception that one's patient outcomes were already optimal; a perceived lack of non-operative alternatives for the management of end-stage osteoarthritis, concerns about mandatory cut-offs for patient-centred care and concerns about the medicolegal implications of using a decision aid.

Conclusions Multifaceted implementation interventions are required to ensure that orthopaedic surgeons are ready, willing and able to use a TKA decision aid. Audit/ feedback to address current decision-making biases such as overconfidence may enhance readiness to uptake. Policy changes and/or incentives may enhance willingness to uptake. Finally, the design/implementation of effective non-operative treatments may enhance ability to uptake by ensuring that surgeons have the resources they need to carry out decisions.

\section{BACKGROUND}

Up to one quarter of total knee arthroplasties (TKA) are performed on inappropriate candidates according to evidence-based criteria $^{1}$
Strengths and limitations of this study

- Very few qualitative studies involving orthopaedic surgeons have been published in the literature.

- This study took place in one of the largest arthroplasty clinics in Australia. All 20 surgeons performing total knee arthroplasty in this hospital participated in a one-to-one interview.

- A theoretical framework was used to systematically explore the barriers and facilitators to uptake of a decision aid by orthopaedic surgeons.

- Consistent findings are documented between this single-site study and international surveys of surgeon' attitudes.

- Beliefs and attitudes are distinct from actual behaviour, and therefore the themes elicited in this study do not provide evidence of the actual influences on uptake of a decision aid.

and a similar proportion experience minimal clinical benefit from surgery. ${ }^{2}$ The rates of TKA are increasing ${ }^{3}$; differentiating who will derive a clinically meaningful improvement from TKA from others is a key challenge for orthopaedic surgeons. While the degree of osteoarthritis (OA) severity, pain severity and the impact of pain are key indicators for TKA, surgeons' consideration of other evidence-based indicators such as psychosocial factors remains varied. ${ }^{4-6}$ Observations of orthopaedic consultations suggest that other 'unstated factors' may also influence clinical judgements such as the surgeons' beliefs in their own ability to conduct surgery and their 'instincts' about the patients' ability to cope with pain. ${ }^{7}$

In recent years, there has been a move towards a model of shared decision making in orthopaedics as part of informed consent. ${ }^{89}$ This model implies that surgeons have a duty to inform patients about the benefits and harms of TKA and the likelihood of their occurrence, supporting them to arrive at an 
informed, shared decision. Evidence suggests that there is often a lack of time during the clinical encounter for patients to consider or discuss all available treatment options and arrive at a decision congruent with their own preferences. ${ }^{10}$

To help address variations in clinical judgements and promote shared decision making, decision aids may be useful. ${ }^{11} 12$ Decision aids can be designed to estimate important, patient-specific risks of responding to surgery, based on independent prognostic correlates of post-TKA response such as body mass index, degree of OA severity, preoperative pain, function and mental health. ${ }^{13}$ Similar aids have been found to predict outcomes in other areas of medicine with superior accuracy to clinical judgements alone. ${ }^{1415}$ Decision aids have improved patient knowledge and confidence in decisions ${ }^{16}$ and have even been found to reduce the rate of surgical procedures. ${ }^{17}$

Orthopaedic surgeons recognise the need for an aid to support their decision making for TKA and optimise communication with patients. ${ }^{6}{ }^{18}$ However, the uptake of decision aids among surgeons has been low. ${ }^{9} \mathrm{~A}$ number of factors can influence uptake of a decision aid, and the success of implementation efforts depends on the careful assessment of the barriers to, and facilitators of, uptake. ${ }^{19}$ The implementation literature advocates the use of theory to ensure the systematic identification of such factors and inform the design of interventions to address them. ${ }^{20}$ Using theory assists in designing studies that are better able to facilitate behaviour change ${ }^{21}$ and provides a basis for better understanding the processes underpinning behaviour change. ${ }^{22}$ To date, studies of decision making among orthopaedic surgeons have been few and atheoretical, ${ }^{6723}$ and the barriers and facilitators of uptake of decision aids for TKA have not been rigorously explored. ${ }^{918}$

\section{METHODS}

\section{Aim and design}

This theoretically informed qualitative study is the first phase of a wider project seeking to design and implement a decision aid into an Australian orthopaedic clinic setting. The aim of this study was to explore the barriers and facilitators to uptake of a TKA decision aid through structured one-to-one interviews with orthopaedic surgeons.

\section{Participants}

All orthopaedic surgeons and registrars performing TKA at one tertiary teaching hospital in Australia were eligible. Eligible candidates received the study details via email from an institutional administration officer and were invited to contact the researchers to arrange an interview at a time and place suitable to them.

\section{Data collection}

The Theoretical Domains Framework (TDF) ${ }^{24}$ was selected as a comprehensive and validated framework for determining barriers and facilitators related to the implementation of best practice and clinicians' behaviour change. The TDF was developed by implementation scientists to synthesise existing behaviour change theories into a single framework. A six-stage consensus approach resulted in the identification of 12 theoretical 'domains' describing possible mediators of behaviour change. ${ }^{24} \mathrm{~A}$ subsequent validation study ${ }^{22}$ revised the original TDF to 14 domains: knowledge, skills, social/professional role and identity, beliefs about capabilities, optimism, beliefs about consequences, reinforcement, intentions, goals, memory, attention and decision processes, environmental context and resources, social influences, emotions and behavioural regulation. The revised TDF, used in this study, has explained implementation problems and informed the development of theory-informed behaviour change interventions. ${ }^{25-27}$

Interview questions were developed for each domain of the TDF with the advice of content experts in the TDF (SF), orthopaedic surgery (MD) and decision-making processes (AS), and in consultation with the literature. ${ }^{28}$ Interviews were prefaced by stating that the researchers planned to develop a decision aid, thus the discussion was centred around a hypothetical decision aid rather than a defined one. In the first part of the interview, questions aimed to elicit current decision-making processes and biases. In the second part, questions aimed to identify beliefs and attitudes towards decision aids and factors that may influence decisions to use one. Table 1 presents the interview schedule.

The interviewer (SB), a female postdoctoral researcher with methodological expertise in qualitative research, had no previous relationship with the participants and no affiliation with the hospital. Data saturation was considered complete when the beliefs and attitudes of all 20 surgeons working in this setting had been elicited. Faceto-face interviews were conducted with 18 participants in a private office; phone interviews were conducted with two participants. Interviews lasted 20-30 min. Nineteen interviews were audio recorded and transcribed verbatim. One participant did not wish the interview to be recorded, therefore hand-written notes were made during the interview. Participant anonymity was ensured at all times. All transcripts were deidentified prior to data analysis. All participants had the opportunity to review the study findings during a presentation at a scheduled surgical meeting. There was consensus agreement with the researchers' interpretations and no adjustments were made to the study themes.

\section{Analysis}

Adopting an implementation approach, ${ }^{26}$ three stages of data analysis were conducted. In the first stage, two researchers (SB and EN) independently coded interview transcripts by classifying each interview response or utterance into one of the 14 TDF domains. For example, this response to the first question in the interview schedule: 'I think 22 per cent is the high end. But there are a lot 


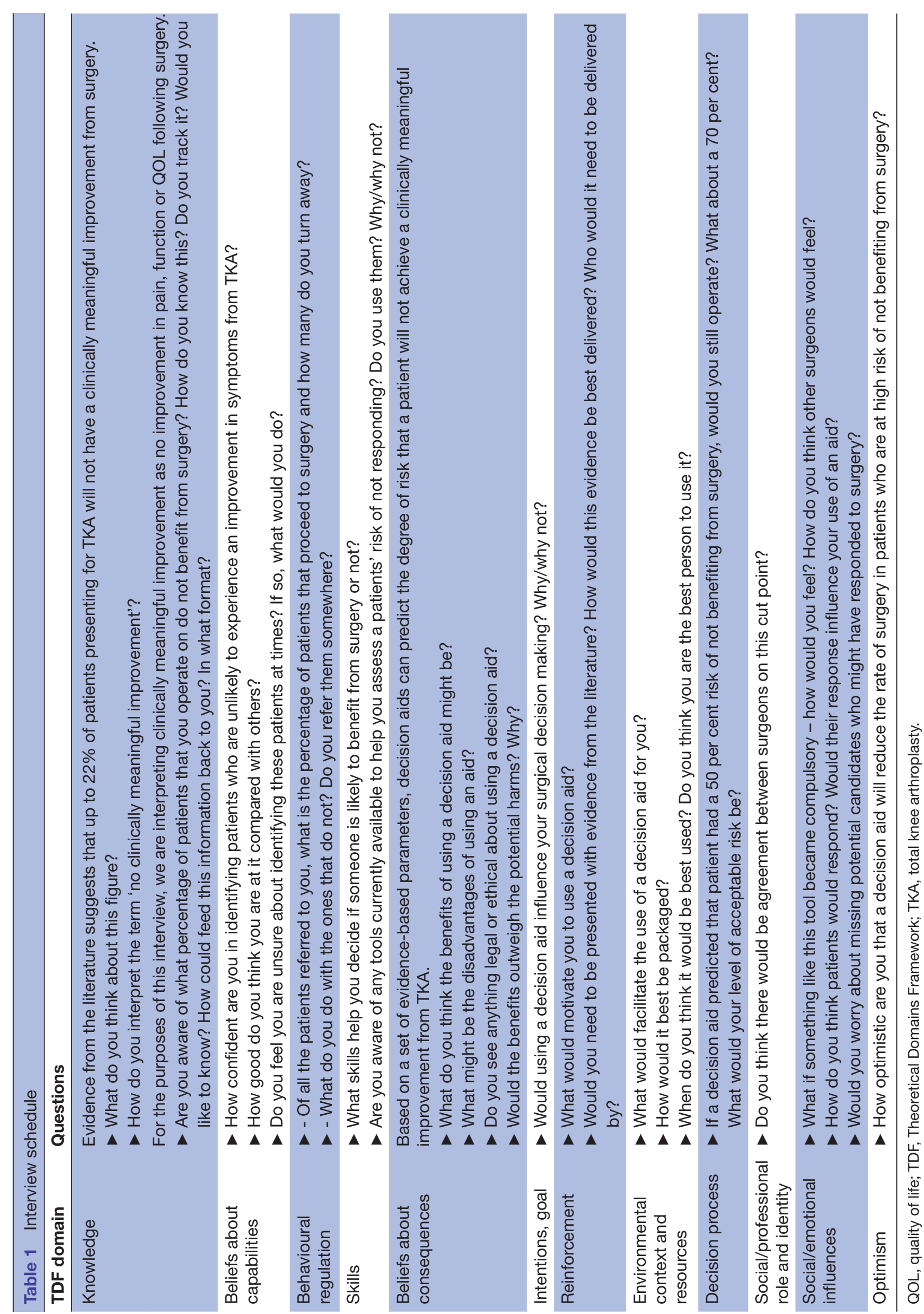


of different papers that all suggest 10, 15, 20 per cent', was classified into the 'Knowledge' domain of the TDF. Definitions for each domain were derived from the literature $^{19}$ and adapted to the study context. Pilot coding was performed in which the two researchers independently coded two transcripts. Intercoder comparisons resulted in the refinement of domain definitions (see online supplementary file). This process was conducted three times, until the two researchers were confident that all relevant interview responses could be clearly coded into one domain. The two researchers then independently coded all 20 transcripts. Disagreements were discussed, and consensus was reached in each instance. Coded responses were uploaded into a qualitative data sorting software $\left(\right.$ Codesort $\left.^{29}\right)$ to facilitate further analysis.

In the second stage of analysis, one researcher (SB) generated 'belief statements' based on the coded interview responses. For example, from the response: 'I think 22 per cent is the high end. But there are a lot of different papers that all suggest 10, 15, 20 per cent' classified in the 'Knowledge' domain, we generated the belief statement: 'I am aware of the literature that up to 20 per cent of patients do not have a clinically meaningful improvement from TKA'. Belief statements were worded such that they could describe similar responses from different participants. Belief statements were reviewed by two further researchers (EN and $\mathrm{MD}$ ), before being interpreted as a likely 'facilitator' or 'barrier' to surgeon's uptake of a decision aid. Continuing the example above, the belief statement: 'I am aware of the literature that up to 20 per cent of patients do not have a clinically meaningful improvement from TKA' was interpreted as a facilitator to uptake, in that we considered surgeons would be more likely to use a decision aid if they were aware that a substantial proportion of TKA's resulted in suboptimal outcomes.

In the third stage of analysis, we identified the domains most likely to influence surgeon's behaviour (ie, using a decision aid or not). This was determined by: (1) frequency of beliefs across transcripts and (2) the perceived strength of beliefs in influencing behaviour. To illustrate, the belief statement: 'I think that the percentage of my patients who achieve clinically meaningful improvement is higher than that reported in the literature' appeared in 17/20 transcripts and was considered to have a strong influence on whether a surgeon would use a decision aid or not, implying that behaviour change (ie, using a decision aid) was unlikely if surgeons considered that their patients' outcomes were already optimised. 'Knowledge' was therefore identified as a relevant domain. Where the researchers considered that beliefs within and between domains represented similar barriers/facilitators, these were grouped into themes. We present frequencies of beliefs (see table 2) to provide the reader with a better understanding of the range of interview responses and to assist us in identifying 'relevant' domains of the TDF. However, readers should be cognisant that the absence of a belief in a transcript is not the same as a lack of endorsement.
RESULTS

\section{Participants}

Aggregate data describing the demographics of the sample is provided to protect individual anonymity. The sample comprised of 15 consultant surgeons and 5 registrars. The surgeons' total experience performing TKA ranged from 6 months to 30 years (mean \pm SD: $12.9 \pm 9.3$ ), and the number of TKAs performed each month ranged from less than 1 to $12($ mean \pm SD: $5.9 \pm 3.0)$.

\section{Relevant domains}

Across the 20 interview transcripts, 628 utterances were coded into the 14 domains. Eleven domains of the TDF were identified as relevant: knowledge, behavioural regulation, memory attention and decision processes, beliefs about capabilities, skills, goals, social/professional role and identity, intention, beliefs about consequences, environmental context and reinforcement. Table 2 presents the relevant domains, with specific belief statements supported by example quotes.

The seven themes are described below, illustrated by interview extracts found in table 3 and denoted in the text as 'Quote' numbers, for example, Q1.

\section{Themes identified}

Knowledge of one's own patient outcomes

(Relevant TDF domains: Goals, Knowledge, Behavioural regulation and Beliefs about capabilities)

The goal of all participations was to optimise outcomes for their patients (Q1). While almost all participants $(n=19)$ were aware of the literature that up to 20 per cent of patients undergoing TKA have no clinically meaningful improvement from surgery, most believed that this percentage was significantly lower in the patients they operated on $(\mathrm{Q} 2)$. All participants based this estimation on patient presentations at postsurgical follow-up appointments. However, participants acknowledged the 'rose-coloured glasses' they saw their patients through, citing the subtleties of rapport that made patients less likely to report dissatisfaction with surgery (Q3).

It was also recognised that patients with poor post-surgical outcomes may be less likely to attend follow-up appointments, choosing to seek care elsewhere. Participants believed that tracking long-term patient outcomes through an existing joint registry could counteract this (Q4 and Q5).

However, a key problem identified by many participants was how to define clinically meaningful improvement. Seven surgeons emphasised the importance of 'asking the right question in the right way' ( $\mathrm{Q} 6$ and $\mathrm{Q} 7)$. The discrepancy between surgeons' perception of clinically meaningful improvement and that of their patients was commonly attributed to mismatched surgeonpatient pre-surgical expectations of TKA. Surgeons reported their expectations of TKA to be a resolution of joint pathology with some corresponding improvement in pain. While disease and pain severity were key considerations in surgical decision making, all participants 


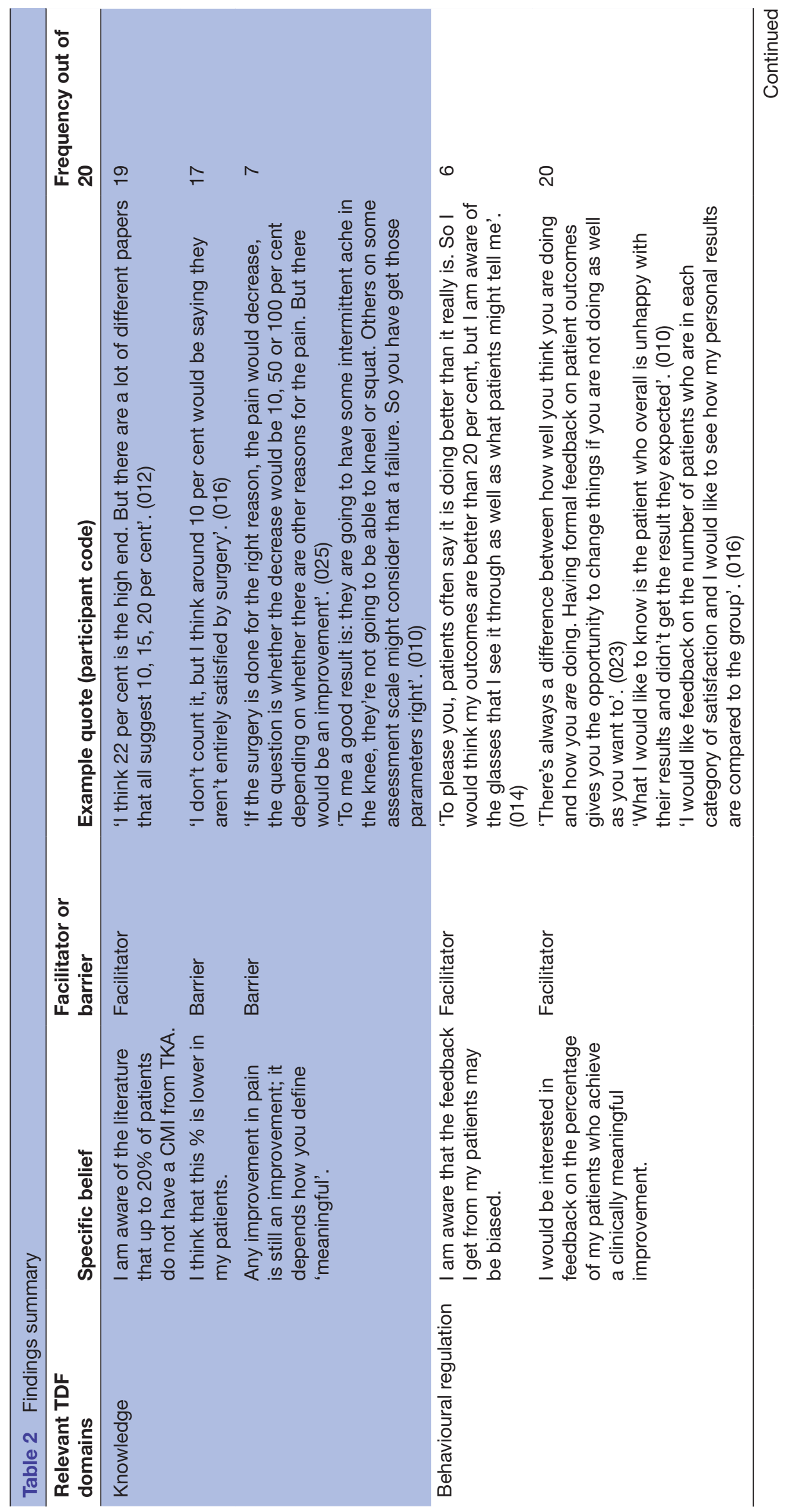




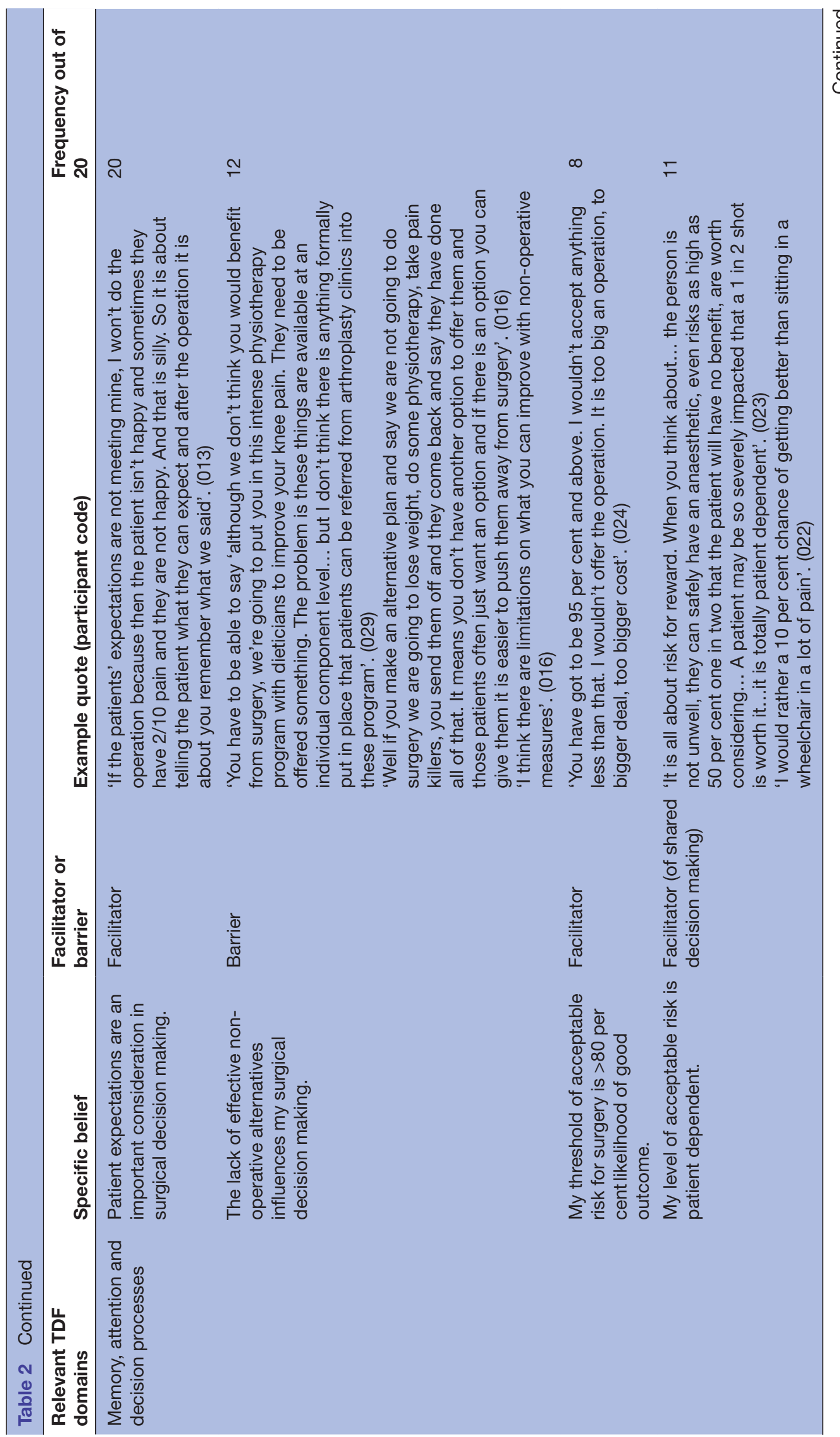




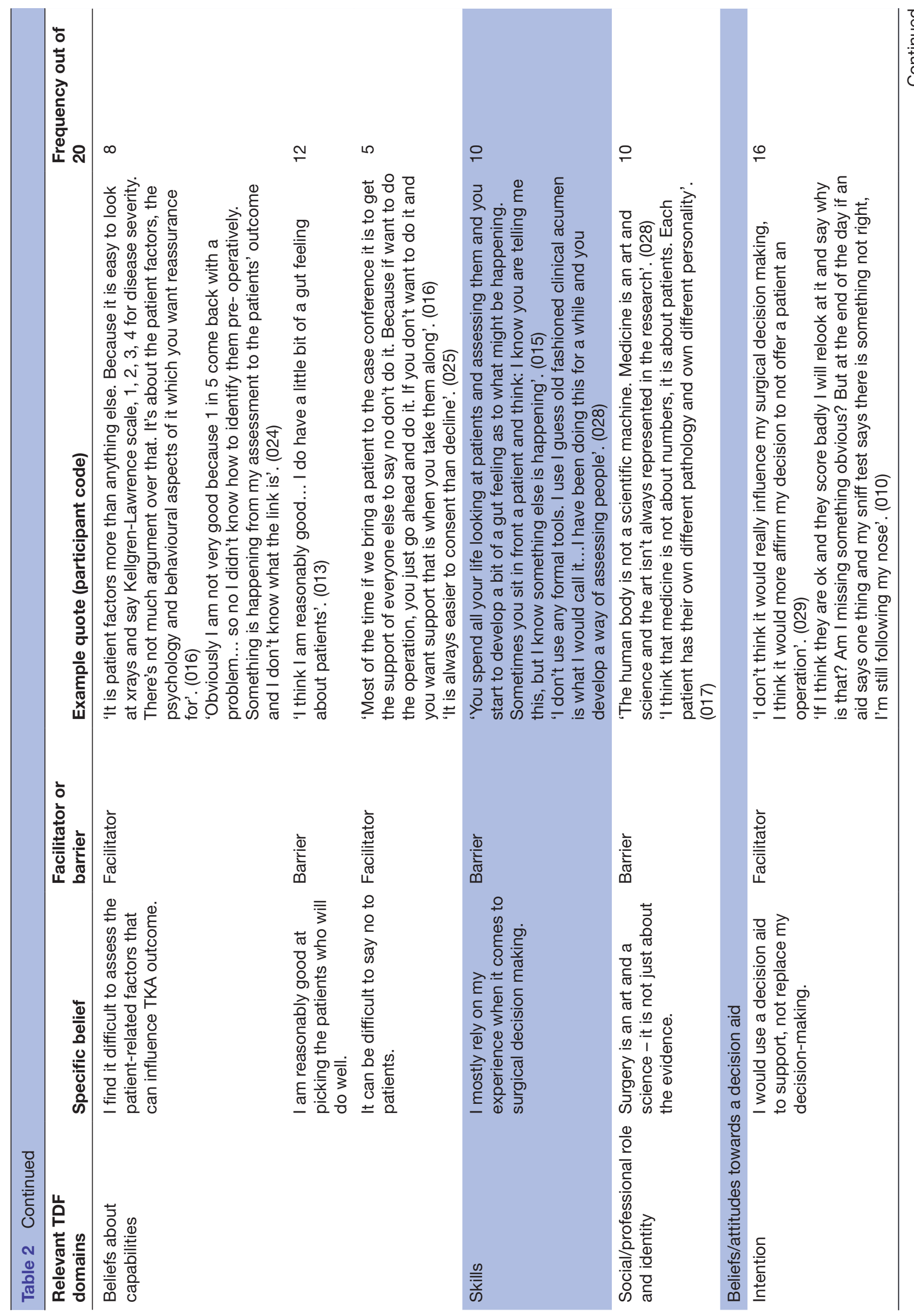




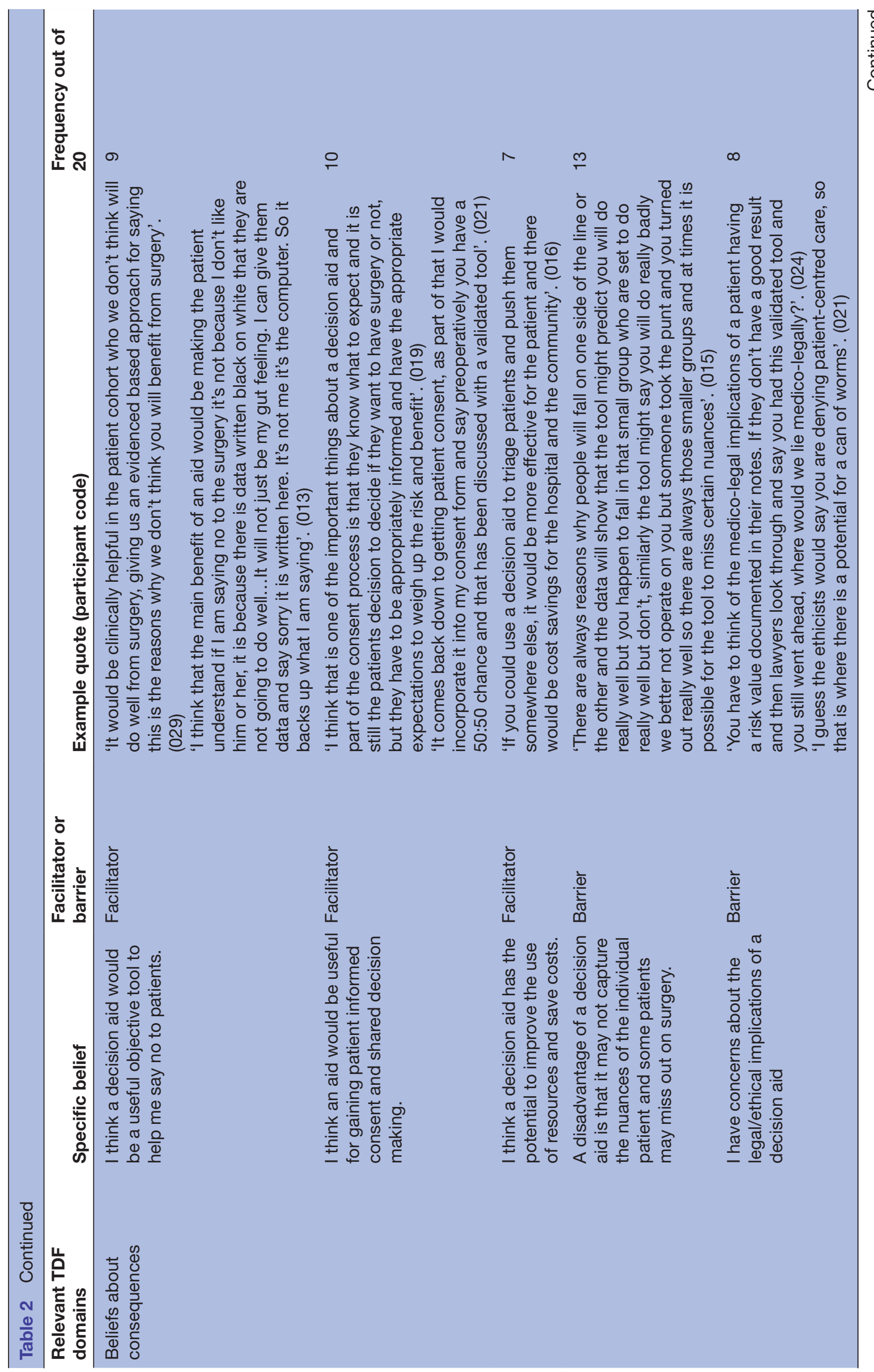




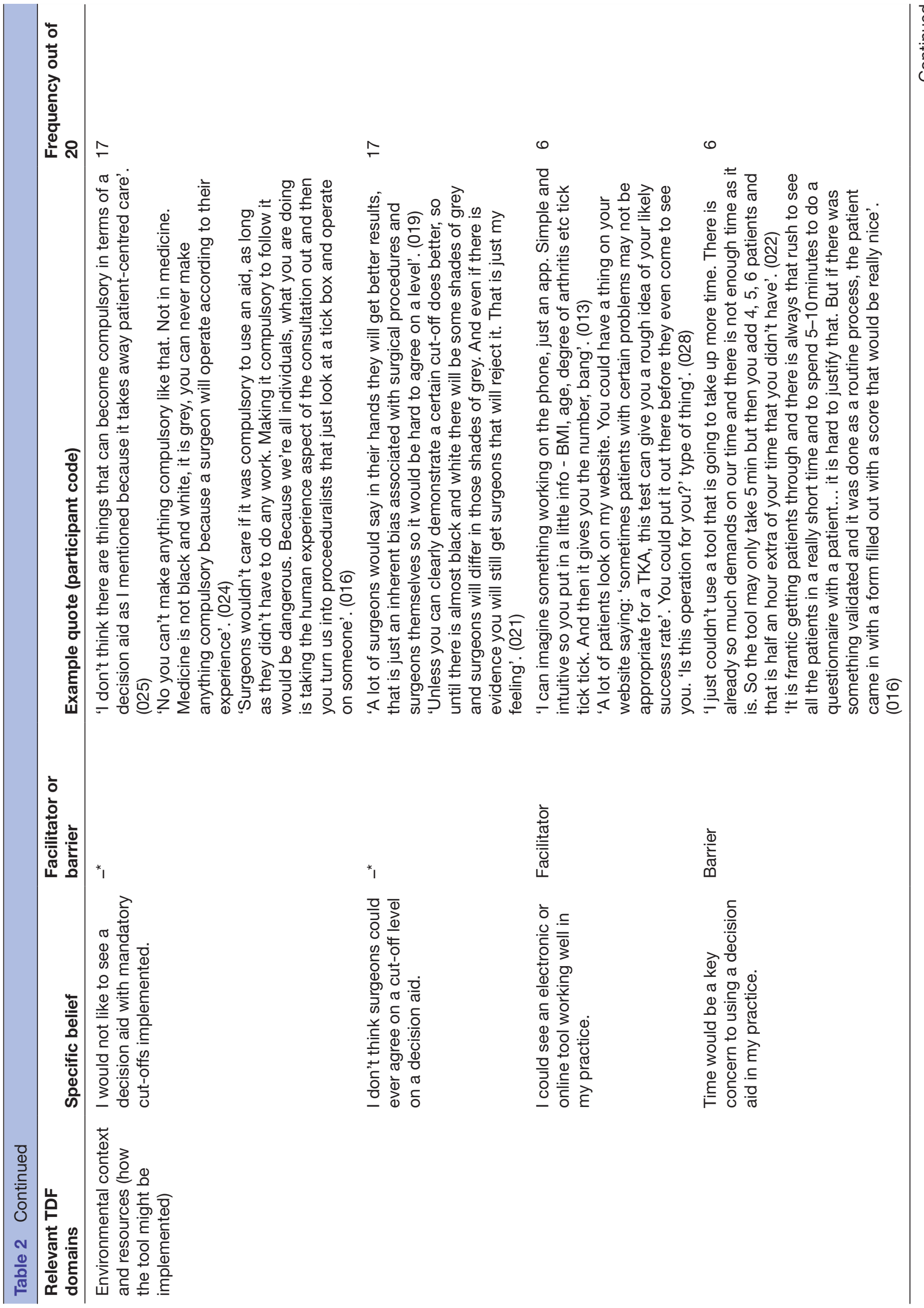




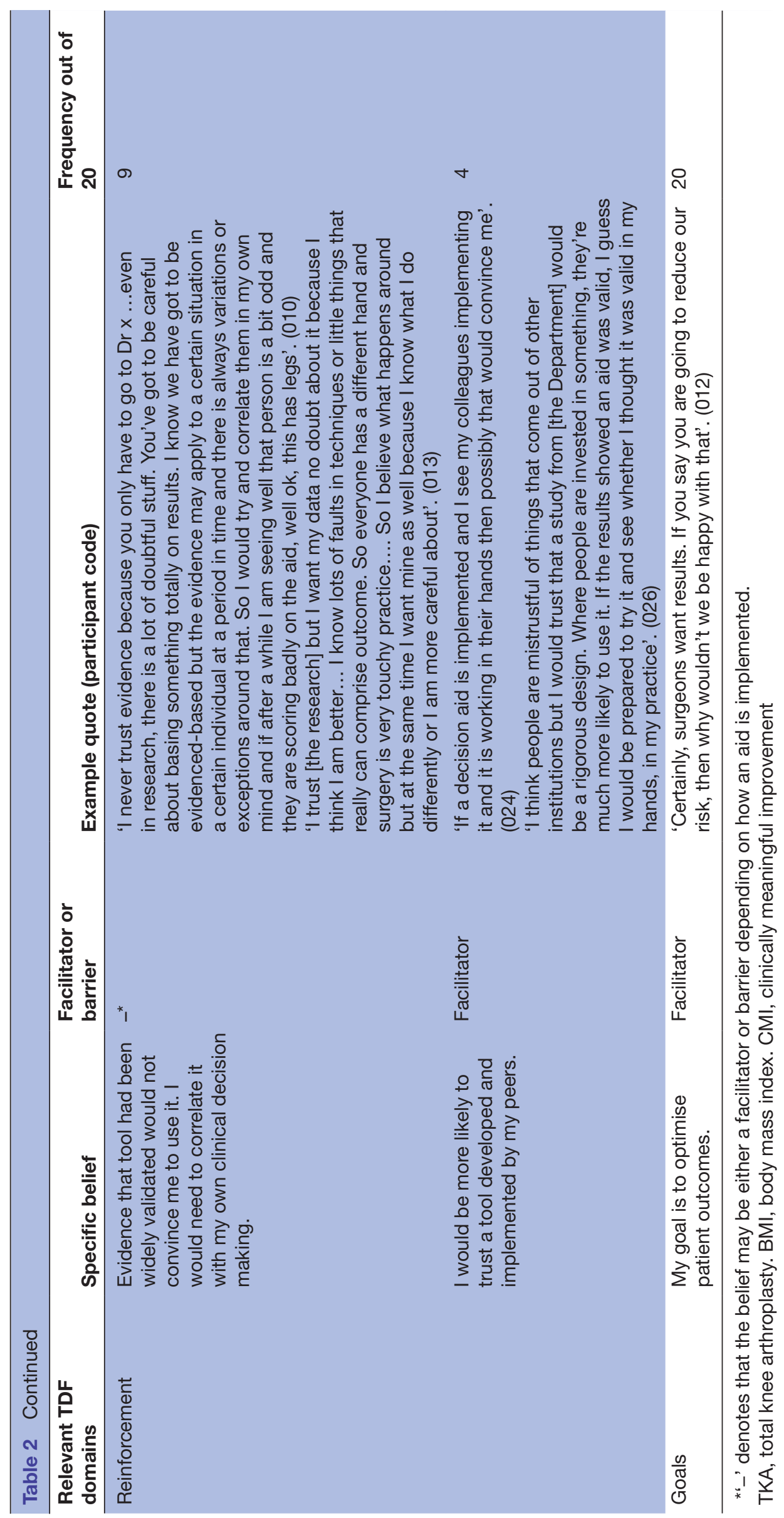




\section{Table 3 Supporting extracts}

\section{Quote number Quote (participant code)}

Q1 'Ultimately, we will always do our best for the patient'. (024)
'I don't count it, but you get an impression. Around 10 per cent of my patients would be saying they are not
entirely satisfied by surgery'. (016)
'Often, to please you, patients say that it is doing better than it really is. So I would think my outcomes are
better than 20 per cent, but I am aware of the glasses that I see it through as well as what patients might tell
me'. (014)

Q4 'There's always a difference between how well you are doing and how well you think you are doing. Having formal feedback on patient outcomes gives you the opportunity to change things if you are not doing as well as you want to'. (023)

Q5 'If patients choose not to come back, the only way you have got to track them is looking at your results from the registry. But I want to know the answers to the clinical questions - are you happy? Is your pain better than it was pre-op? How you ask the question matters'. (028)

Q6 'If you received feedback that the rate of clinically meaningful improvement reported by your patients is not as high as you think it should be, you have to look at whether you are not picking the right patients, or you are operating on patients that are not going to do well. I think it would be more likely to be the way the question is asked. I would want to check who is asking the questions, what they are asking and how they are asking it'. (023)

\begin{tabular}{|c|c|}
\hline Q7 & $\begin{array}{l}\text { 'To me a good result is: they are going to have some intermittent ache in the knee, they are not going to be } \\
\text { able to kneel or squat, they are going to be aware that it is there. That to me is a good result. Now others on } \\
\text { some assessment scale they might say well that is in our system considered a failure thing, so you have get } \\
\text { those parameters right'. (010) }\end{array}$ \\
\hline Q9 & $\begin{array}{l}\text { 'If the patients' expectations are not meeting mine, I won't do the operation because then the patient isn't } \\
\text { happy and sometimes they have } 2 / 10 \text { pain and they are not happy'. (013) }\end{array}$ \\
\hline Q11 & $\begin{array}{l}\text { 'You spend all your life looking at patients and assessing them and you start to develop a bit of a gut feeling } \\
\text { as to what might be happening when you sit in front of a patient and you might be saying you know you are } \\
\text { telling me this but actually I know something else is happening'. (015) }\end{array}$ \\
\hline Q13 & $\begin{array}{l}\text { 'If I think they are OK and they score badly I will relook at it and say why is that? Am I missing something } \\
\text { obvious? But at the end of the day if the tool says one thing and my sniff test says there is something not } \\
\text { right, I am still following my nose'. (010) }\end{array}$ \\
\hline Q14 & $\begin{array}{l}\text { 'Not every tool is perfect and it may not capture every patient... the danger is we may end up refusing to do } \\
\text { something because of this tool and therefore the patient may not receive the appropriate treatment based on } \\
\text { a decision aid and nothing is } 100 \text { per cent so you have to expect some patients would fall through the cracks'. } \\
\text { (019) }\end{array}$ \\
\hline Q15 & $\begin{array}{l}\text { 'I think people are mistrustful of things that come out of other institutions but I would trust that a study from } \\
\text { [the Department] would be a rigorous design. Where people are invested in something, they are much more } \\
\text { likely to use it. If the results showed the tool was valid, I guess I would be prepared to try it and see whether I } \\
\text { thought it was valid in my hands, in my practice'. (026) }\end{array}$ \\
\hline
\end{tabular}


Table 3 Continued

\section{Quote number Quote (participant code)}

Q18 'It comes back down to getting patient consent, as part of that I would incorporate it into my consent form and say preoperatively you have a 50:50 chance and that has been discussed with a validated tool. If the patient wishes to go ahead, they can make that informed decision'. (021)

\begin{tabular}{|c|c|}
\hline Q19 & 'A patient may be so severely impacted that a 1 in 2 shot is worth it... it is totally patient dependent'. (023) \\
\hline Q20 & $\begin{array}{l}\text { 'You have got to be } 95 \text { per cent and above. I wouldn't accept anything less than that. I wouldn't offer the } \\
\text { operation. It is too big an operation, too big a deal, too big a cost'. (024) }\end{array}$ \\
\hline Q21 & $\begin{array}{l}\text { 'You have to think the medico-legal implications of a patient having a risk value documented in their notes. If } \\
\text { they don't have a good result and then some have the lawyers look through and say you had this tool that was } \\
\text { validated and you still went ahead where would we lie medico-legally?'. (024) }\end{array}$ \\
\hline Q22 & $\begin{array}{l}\text { 'I guess the ethicists would say you are denying patient-centred care, so that is where there is a potential for a } \\
\text { can of worms'. (021) }\end{array}$ \\
\hline Q23 & 'I don’t think it can become compulsory because it takes away patient-centred care'. (025) \\
\hline Q24 & $\begin{array}{l}\text { 'If you could use the tool to triage patients and push them some where else, it would be more effective for the } \\
\text { patient and there would be cost savings for the hospital and the community'. (016) }\end{array}$ \\
\hline Q25 & $\begin{array}{l}\text { 'You have to be able to say: "although we don't think you would benefit from surgery, we are going to put you } \\
\text { in this intense physiotherapy program with dieticians and this is how we are going to improve your knee pain'" } \\
\text { They need to be offered something. The problem is these things are available at an individual component level } \\
\text { - we have got dieticians and physiotherapists and exercise groups, but I don't think there is anything formally } \\
\text { put in place that patients can be referred from arthroplasty clinics into these program'. (029) }\end{array}$ \\
\hline Q26 & $\begin{array}{l}\text { 'A lot of surgeons would say in their hands they will get better results, that is just an inherent bias associated } \\
\text { with surgical procedures and surgeons themselves so it would be hard to agree on a level'. (019) }\end{array}$ \\
\hline Q27 & $\begin{array}{l}\text { 'Well compulsory to have it? Ok. That would be easy to do and surgeons wouldn't care as long as they didn't } \\
\text { have to do any work. Making it compulsory to follow it would be dangerous. Because we are all individuals, } \\
\text { what you are doing is taking the human experience aspect of the consultation out and then you turn us into } \\
\text { proceeduralists that just look at a tick box and operate on someone'. (016) }\end{array}$ \\
\hline Q28 & $\begin{array}{l}\text { 'I can imagine something working on the phone, an app. It needs to be simple and intuitive - so you put in a } \\
\text { little info - BMI, age, degree of arthritis etc... tick tick tick. And then it gives you the number, bang'. (013) }\end{array}$ \\
\hline Q29 & $\begin{array}{l}\text { 'I think it is something that should be done by the surgeon. It is also part of the process where the surgeon } \\
\text { gets to know the patient as well - not just their xrays and physical examination but also their psychosocial } \\
\text { situation'. (019) }\end{array}$ \\
\hline Q30 & $\begin{array}{l}\text { 'I would want the tool to be applied within the consultation. Because I would never believe a value until I have } \\
\text { seen the person. Because we might just have one of those weird situations that fall out of the 'normal' range'. } \\
\text { (010) }\end{array}$ \\
\hline Q31 & $\begin{array}{l}\text { 'When you have got } 10 \text { minutes for a consultation you don't have time to spend another } 10 \text { minutes going } \\
\text { through the tool. So it would have to be either the patient themselves or secretarial person prior to the } \\
\text { consultation'. (012) }\end{array}$ \\
\hline Q32 & $\begin{array}{l}\text { 'I have a lot of patients look me up on my website. You could have a thing on your website saying: 'sometimes } \\
\text { patients with certain problems may not be appropriate for a TKA, this test can give you a rough idea of your } \\
\text { success rate'. You could put it out there before they even come to see you. 'Is this operation for you?' type of } \\
\text { thing'. (028) }\end{array}$ \\
\hline
\end{tabular}

acknowledged that patient expectations were important (Q8 and Q9).

Reliance on 'clinical intuition'

(Relevant TDF domains: Memory, attention and decision processes and Skills)

The accurate assessment of patient expectations presented a challenge for many surgeons. While the physical aspects of the clinical assessment were routine skills that all participants believed they had mastered well, many junior and senior surgeons reported difficulties assessing the psychological aspects of the patients' presentation (Q10).
Only a few participants were aware of any validated tools to assess pre-surgical patient predictive factors. One participant had prior knowledge of a decision aid but had not used it. None of the other participants were aware of any decision aid for TKA. Participants relied on their 'clinical intuition' for patients who were less likely to do well. A 'gut-feeling' for patients was developed with experience over time $(\mathrm{Q} 11)$.

The role of aids in supporting clinical decision making (Relevant TDF domains: Intention and Reinforcement)

Fifteen of the participants believed an aid would support decision making, like a 'barometer' or weather 
app to forecast outcomes, particularly in patients they were unsure about (Q12, Q13). However, most participants $(n=13)$ believed an aid would be insensitive to nuances at an individual level and could therefore not replace their clinical acumen (Q14).

All participants expected to be provided with evidence that a decision aid had been rigorously validated and shown to have high specificity and sensitivity before considering using it. Participants were more likely to trust this evidence if it came from their own institution (Q15). However, half of the participants reported that evidence supporting the validity of a decision aid would not be sufficient to convince them to use it. Instead they would need to see how the tool correlated with their own clinical decision making (Q16).

Implications of a decision aid for patient-surgeon communication and shared decision making

(Relevant TDF domains: Beliefs about consequences and Memory attention and decision processes)

Nine participants perceived that a decision aid would give them an evidence-based approach for saying 'no' to patient, particularly for those participants who reported difficulty declining for surgery (Q17). Many participants $(\mathrm{n}=10)$ believed an aid could be an important component of patient informed consent, providing patients with objective data on their likely risk outcomes, enabling them to have the appropriate expectations to weigh up the risk and benefits of surgery for themselves. In this way, a decision aid was seen as a valuable support to shared decision making (Q18).

When asked how they would feel about operating if a decision aid predicted a patient had a 50 per cent chance of not responding to TKA, surgeons were divided in their responses. While 11 believed that decisions to proceed would need to be shared with the patient (Q19), eight reported they would not consider surgery unless there was a greater than $80 \%$ per cent chance the patient would respond $(\mathrm{Q} 20)$.

\section{Ethical and legal concerns about decision aids}

(Relevant TDF domain: Beliefs about consequences)

Eight participants had medicolegal concerns about documenting a specific risk value in patient records, with a few believing that such information would have to be deliberately withheld from patients in the case it fell into the 'wrong' hands (Q21). While some participants believed it would be unethical not to use a decision aid if it had been shown to improve patient outcomes, others were concerned about the ethical implications of a tool if imposed cut-offs were used to deny patients' surgery (Q22 and Q23).

\section{Available resources and organisational culture as barriers to} uptake

(Relevant TDF domains: Environmental context and resources, Beliefs about consequences and Social/professional role and identity)
Almost all participants expressed concerns about making an aid compulsory and imposing mandatory cut-off levels. While many recognised that implementing the tool in this way would have the potential to improve the use of valuable health resources and save costs (Q24), several participants commented that mandatory cut-offs would only be possible if an effective, non-operative alternative was made available for patients that were denied surgery. The existing lack of effective alternative to surgery was seen as a key barrier (Q25).

There was widespread agreement among surgeons $(n=17)$ that the logistics of agreeing on a cut-off value for acceptable risk, and the threat to surgeons' professional identity as a patient-centred practitioner, were insurmountable barriers to imposing mandatory cut-offs (Q26 and Q27).

\section{Format and content of a decision aid}

(Relevant TDF domain: Environmental context and resources)

Finally, physically integrating an aid into clinical practice was not seen as a key barrier to implementation. An electronic or online format was seen as the most likely way an aid could be implemented, particularly given the strict time constraints placed on outpatient clinics (Q28).

Most believed that an aid would be best used within the patient-surgeon consultation (Q29 and Q30), while a couple suggested that an aid could be designed for patients to use on their own or with a support network to save time in the clinical consultation (Q31 and Q32).

\section{Irrelevant domains}

Three domains did not appear to have a salient influence on the target behaviour. These were: Optimism, Emotion and Social influences. Optimism was closely connected to the domain reinforcement; that is, surgeons reported being neither optimistic nor pessimistic about the utility of an aid until they had seen evidence of its effectiveness. The Emotion domain did not appear relevant as described by one surgeon: 'I am not worried about the implications of a tool. TKA is wellness surgery, in the worst case scenario they don't get a new knee'. The Social influence of patients did not appear relevant as surgeons believed patients were accustomed to filling out questionnaires. While surgeons were more likely to trust an aid if it had been developed by their peers, they preferred to test the tool 'in their own hands' and therefore the social influences of other surgeons using an aid appeared limited.

\section{DISCUSSION}

These findings have implications for the future design and implementation of decision aids into surgical clinical practice. It is possible that current decision-making biases may be key barriers to uptake. Surgical decision making involves the consideration of the risks versus benefits of surgery. ${ }^{30}$ In this study, participants expressed confidence 
in their ability to successfully remove diseased tissue and correct joint deformity, and the likelihood of some corresponding improvement in pain severity was perceived to be high. At the same time, participants perceived that the risk their patients would not respond to TKA was low. One potential explanation for this is an apparent lack of clarity around the definition of 'clinically meaningful improvement' and 'non-response'. The literature suggests that patients' perceptions of 'clinically meaningful improvement' depend on their satisfaction with improvements in pain and function, closely linked to their expectations of surgery. ${ }^{31}$ In contrast, the surgeons in this study believed that a resolution in joint pathology and some corresponding improvement pain could be considered 'meaningful improvement'. This lack of clarity is important, as surgeons who believe that their patient outcomes are already optimised may be less motivated to use a decision aid.

Another explanation may be that surgeons in this sample exhibited an overconfidence bias. Indeed, most participants believed their own patient outcomes were better than that reported in the literature. While it is possible that this belief is accurate and outcomes among this sample of surgeons are indeed above average, similar observations were made in a survey study involving 700 orthopaedic surgeons in Europe and North America. ${ }^{32}$ In that study, 83 per cent of surgeons surveyed considered themselves to be above-average diagnosticians, 74 per cent believed they were above average surgeons and 25 per cent believed they were in the top 5 per cent of surgeons. ${ }^{32}$ This overconfidence bias may lead to a confirmation bias in which surgeons only notice things that agree with their point of view and are less attentive to alternative viewpoints due to cognitive dissonance. ${ }^{33} \mathrm{~A}$ confirmation bias can have consequences for the uptake of a decision aid as surgeons may be less likely to consider evidence that contradicts their clinical experience.

Surgical decision making also involves weighing up of the risks versus benefits of non-operative interventions. ${ }^{30}$ The participants highlighted a lack of effective non-operative interventions for end-stage knee OA. A study among Dutch orthopaedic surgeons similarly documented a lack of confidence in the efficacy of non-surgical treatments associated with a decreased referral rate. ${ }^{34}$ Indeed, there is currently a dearth of evidence-based, non-operative interventions for patients with end-stage knee OA either as an alternative to surgery or as a definitive management when the patient is deemed unsuitable for TKA. In the absence of a treatment alternative, surgery with all its risks and costs is often the default intervention. This is an important barrier to uptake as evidence suggests surgeons will not use an aid if they do not have the resources to carry out the decisions ${ }^{28}$ or if the aid does not support their own view about effective treatments. ${ }^{35}$

The participants in this study believed a decision aid could promote shared decision making and enhance informed consent for TKA. Similar attitudes towards decision aids were identified in a previous review ${ }^{9}$ and a survey of the wider population of orthopaedic surgeons. ${ }^{18}$ It may be that surgeons can be motivated to uptake a decision aid, because it is the ethical thing to do. ${ }^{36}$ However, our study identified concerns about the ethical implications of imposing mandatory cut-offs that would determine eligibility for TKA. These concerns may reflect the participants' desire to defend professional discretion and autonomy. We take the opportunity to emphasise the function of a decision aid is to support, not replace, clinical decision making. However, concerns were also expressed about the legal implications of using a decision aid. This finding contrasts with a study involving US orthopaedic surgeons who believed decision aids may reduce litigation and malpractice insurance premiums by enhancing shared decision making. ${ }^{37}$ It may be that these concerns are unique to the medicolegal context of Australia. Certainly, surgeons will be less likely to be uptake a tool if they perceive it makes them vulnerable to medicolegal action.

\section{Implications}

The Ready, Willing and Able model posits that these three preconditions must be satisfied before a decision aid will be adopted. ${ }^{38}$ 'Ready' refers to the perceived benefit of changing the status quo and adopting an aid to support decision making. 'Willing' refers to the perceived legitimacy of a decision aid and a willingness to overcome objections and concerns. 'Able' refers to the ability to adopt the decision aid given available resources. ${ }^{39}$ Implications for enhancing the readiness, willingness and ability of Australian orthopaedic surgeons to uptake a TKA decision aid are described below.

To enhance readiness to uptake, current decision-making biases may need to be addressed. Information to counter these biases could be provided through audit and feedback. Audit and feedback is defined as a 'summary of the clinical performance of healthcare provider(s) over a specified period of time' (Ivers et al, p5) ${ }^{40}$ It may be particularly effective when clinicians' ability to accurately self-assess is limited. ${ }^{41}$ This assumes that clinicians are motivated to improve care but lack intention to change current behaviour because they are unaware of their suboptimal performance. While effect sizes may depend on the format and context in which feedback is provided, findings from a Cochrane review suggest that audit and feedback can yield important improvements in professional practice. ${ }^{40}$ Clarity and consensus on the definition of 'clinically meaningful improvement' and 'non-response' to TKA is likely to influence the success of an audit and feedback intervention in this context. Future research is needed to explore how audit and feedback is best delivered in this context including the immediacy and frequency of feedback, and the potential to incorporate other approaches such as the use of surgeon incentives. ${ }^{42}$

To enhance willingness to uptake, an audit and feedback intervention before and after using a decision aid may be necessary for orthopaedic surgeons to gain confidence in 
its legitimacy. The findings suggest that validating a decision aid in large multicentre trials may not be sufficient to encourage uptake, as surgeons believed such trials do not account for variations in case load and years of experience. Surgeons perceived themselves as scientists and 'artists', believing that surgical success depended, in part, on the 'hands' that performed it. This suggests that orthopaedic surgeons may need to be given the opportunity to validate a decision aid 'in their own hands'.

Addressing concerns about the medicolegal implications of a decision aid may also be necessary to enhance willingness to uptake. This may require the consultation of multiple stakeholders including policy makers, lawyers and institutional administration before the implementation of a decision aid. Legislative changes in the USA have recognised decision aids as a higher standard of informed consent, ${ }^{8}$ and in the UK, incentives to use decision aids are being developed. ${ }^{9}$ There is evidence that mandating the use of a decision aid as a requirement for gaining informed consent results in significantly improved patient outcomes including a reduction in the rate of TKA by 38 per cent in a 6 -month period. ${ }^{43}$ Future studies are required to explore if policy changes and/or incentives might influence willingness to uptake a decisions aid in the Australian context.

To enhance ability to uptake, it appears important to address the dearth of non-operative alternatives to surgery for end-stage knee OA so that surgeons have the resources to carry out decisions. Future research to design and implement effective, evidence-based, non-operative interventions should be a priority. Targeting orthopaedic surgeons' beliefs about the efficacy of such interventions through education strategies will also be important. The study findings suggest that ability to uptake may be enhanced if a decision aid is packaged in an electronic or online format that is quick and easily accessible. Focus groups involving surgeons and patients to gain feedback on prototypes of a decision aid during the design phase is important to ensure that the format and the content are accessible to a range of end users.

\section{Limitations}

Qualitative studies involving orthopaedic surgeons are rare. The strength of this study is the 100 per cent participation rate by orthopaedic surgeons in one tertiary hospital setting. While the sampling strategy means the generalisability of these findings to other contexts may be limited, we have documented significant similarities with international studies. We acknowledge that while beliefs, attitudes and intentions can predict behaviours with a degree of accuracy, they are distinct from actual behaviour. ${ }^{44}$ Thus, the themes elicited in this study do not provide evidence of the actual influences on uptake of a decision aid.

\section{CONCLUSIONS}

Using a theoretical framework to systematically explore barriers and facilitators to uptake, this study will inform the design and implementation of future TKA decision aids. ${ }^{13}$ The findings suggest that a multifaceted approach will be required to ensure that orthopaedic surgeons are ready, willing and able to use a decision aid that can reduce the suffering and economic burden of 'failed' TKA. Research exploring patients' beliefs and attitudes towards a TKA decision aid is required prior to implementation.

Acknowledgements We acknowledge Pascal Buenzli for assistance with the data management software and inter-rater reliability calculation. We extend our thanks to all the surgeons for kindly donating their time to participate in this study.

Contributors SB: led the study design, data collection, data analysis and manuscript preparation. EN contributed to the data analysis. AS, PC and MD contributed to the study design, data analysis and manuscript preparation. SF contributed to the study design and manuscript preparation.

Funding This study was funded by the University of Melbourne FBE/MDHS Collaboration Seed Funding Grant and by the National Health \& Medical Research Council (APP1122983). A/Prof Dowsey holds an National Health \& Medical Research Council Career Development Fellowship (APP1122526).

Competing interests None declared.

Ethics approval This study was carried out in compliance with the Helsinki Declaration. Approval was given by St Vincent's Hospital Melbourne, Australia (QA 019/_16).

Provenance and peer review Not commissioned; externally peer reviewed.

Data sharing statement Requests regarding the datasets used in this study can be directed to the first author. Full interview transcripts will not be shared to protect the anonymity of the study participants.

Open Access This is an Open Access article distributed in accordance with the Creative Commons Attribution Non Commercial (CC BY-NC 4.0) license, which permits others to distribute, remix, adapt, build upon this work non-commercially, and license their derivative works on different terms, provided the original work is properly cited and the use is non-commercial. See: http://creativecommons.org/ licenses/by-nc/4.0/

C Article author(s) (or their employer(s) unless otherwise stated in the text of the article) 2017. All rights reserved. No commercial use is permitted unless otherwise expressly granted.

\section{REFERENCES}

1. Cobos R, Latorre A, Aizpuru F, et al. Variability of indication criteria in knee and hip replacement: an observational study. BMC Musculoskelet Disord 2010;11:249.

2. Dowsey MM, Smith AJ, Choong PFM. Latent class growth analysis predicts long term pain and function trajectories in total knee arthroplasty: a study of 689 patients. Osteoarthritis Cartilage 2015;23:2141-9.

3. Australian Orthopaedic Association. Annual report. Adelaide: National Joint Replacement Registry, 2014.

4. Riddle DL, Jiranek WA, Hayes CW. Use of a validated algorithm to judge the appropriateness of total knee arthroplasty in the United States: a multicenter longitudinal cohort study. Arthritis Rheumatol 2014;66:2134-43.

5. Curtis AJ, Wolfe R, Russell CO, et al. Determining priority for joint replacement: comparing the views of orthopaedic surgeons and other professionals. Med J Aust 2011;195:699-702.

6. Frankel L, Sanmartin C, Hawker G, et al. Perspectives of orthopaedic surgeons on patients' appropriateness for total joint arthroplasty: a qualitative study. J Eval Clin Pract 2016;22:164-70.

7. Gooberman-Hill R, Sansom A, Sanders CM, et al. Unstated factors in orthopaedic decision-making: a qualitative study. BMC Musculoskelet Disord 2010;11:213.

8. Kuehn BM. States explore shared decision making. JAMA 2009;301:2539-41.

9. Jayadev C, Khan T, Coulter A, et al. Patient decision aids in knee replacement surgery. Knee 2012;19:746-50.

10. van den Bemt BJ, Zwikker HE, van den Ende $\mathrm{CH}$. Medication adherence in patients with rheumatoid arthritis: a critical appraisal of the existing literature. Expert Rev Clin Immunol 2012;8:337-51. 
11. Feldman-Stewart D, Brennenstuhl S, Mclssac K, et al. A systematic review of information in decision aids. Health Expect 2007;10:46-61.

12. Sacks GD, Dawes AJ, Ettner SL, et al. Impact of a risk calculator on risk perception and surgical decision making: a randomized trial. Ann Surg 2016;264:889-95.

13. Dowsey MM, Spelman T, Choong PF. Development of a prognostic nomogram for predicting the probability of nonresponse to total knee arthroplasty 1 year after surgery. J Arthroplasty 2016;31:1654-60.

14. Barsoum WK, Murray TG, Klika AK, et al. Predicting patient discharge disposition after total joint arthroplasty in the United States. J Arthroplasty 2010;25:885-92.

15. Kattan MW. Nomograms are difficult to beat. Eur Urol 2008;53:671-2.

16. Stacey D, Bennett C, Barry M, et al. Decision aids for people facing health treatment or screening decisions. Cochrane Database Syst Rev 2011;10:CD001431.

17. Knops AM, Legemate DA, Goossens A, et al. Decision aids for patients facing a surgical treatment decision: a systematic review and meta-analysis. Ann Surg 2013;257:860-6.

18. Adam JA, Khaw FM, Thomson RG, et al. Patient decision aids in joint replacement surgery: a literature review and an opinion survey of consultant orthopaedic surgeons. Ann R Coll Surg Engl 2008;90:198-207.

19. Phillips CJ, Marshall AP, Chaves NJ, et al. Experiences of using the theoretical domains framework across diverse clinical environments: a qualitative study. J Multidiscip Healthc 2015;8:139-46.

20. Eccles M, Grimshaw J, Walker A, et al. Changing the behavior of healthcare professionals: the use of theory in promoting the uptake of research findings. J Clin Epidemiol 2005;58:107-12.

21. Noar SM, Zimmerman RS. Health Behavior Theory and cumulative knowledge regarding health behaviors: are we moving in the right direction? Health Educ Res 2005;20:275-90.

22. Cane J, O'Connor D, Michie S. Validation of the theoretical domains framework for use in behaviour change and implementation research. Implement Sci 2012;7:37.

23. Sansom A, Donovan J, Sanders C, et al. Routes to total joint replacement surgery: patients' and clinicians' perceptions of need. Arthritis Care Res 2010;62:1252-7.

24. Michie S, Johnston M, Abraham C, et al. Making psychological theory useful for implementing evidence based practice: a consensus approach. Qual Saf Health Care 2005;14:26-33.

25. Dobson F, Bennell KL, French SD, et al. Barriers and facilitators to exercise participation in people with hip and/or knee osteoarthritis. Am J Phys Med Rehabil 2016;95:1-89.

26. French SD, Green SE, O'Connor DA, et al. Developing theoryinformed behaviour change interventions to implement evidence into practice: a systematic approach using the Theoretical Domains Framework. Implement Sci 2012;7:38.

27. Patey AM, Islam R, Francis JJ, et al. Anesthesiologists' and surgeons' perceptions about routine pre-operative testing in low-risk patients: application of the Theoretical Domains Framework (TDF) to identify factors that influence physicians' decisions to order preoperative tests. Implement Sci 2012;7:52.

28. Sepucha KR, Mulley AG. Extending decision support: preparation and implementation. Patient Educ Couns 2003;50:269-71.

29. Buenzli P. Codesort software. 2012.

30. Sacks GD, Dawes AJ, Ettner SL, et al. Surgeon perception of risk and benefit in the decision to operate. Ann Surg 2016;264:896-903.

31. Hawker G, Bohm ER, Conner-Spady B, et al. Perspectives of Canadian stakeholders on criteria for appropriateness for total joint arthroplasty in patients with hip and Kknee osteoarthritis. Arthritis Rheumatol 2015;67:1806-15.

32. Teunis T, Janssen S, Guitton TG, et al. Do orthopaedic surgeons acknowledge uncertainty? Clin Orthop Relat Res 2016;474:1360-9.

33. Kahneman D. Thinking, fast and slow. New York, USA: Macmillan, 2011.

34. Hofstede SN, Marang-van de Mheen PJ, Vliet Vlieland TP, et al. Barriers and facilitators associated with non-surgical treatment use for osteoarthritis patients in orthopaedic practice. PLoS One 2016;11:e0147406.

35. Elwyn G, Rix A, Holt T, et al. Why do clinicians not refer patients to online decision support tools? Interviews with front line clinics in the NHS. BMJ Open 2012;2:e001530.

36. Tai-Seale M, Elwyn G, Wilson CJ, et al. Enhancing shared decision making through carefully designed interventions that target patient and provider behavior. Health Aff 2016;35:605-12.

37. Weinstein JN, Clay K, Morgan TS. Informed patient choice: patient-centered valuing of surgical risks and benefits. Health Aff 2007:26:726-30.

38. Lesthaeghe R, Ready VC. willing and able: A conceptualization of transitions to new behavioral forms. In: Casterline J, ed. Diffusion processes and fertility transition: selected perspectives. Washington DC: National Academies Press, 2001:240-64.

39. Shultz CG, Jimbo M. Decision aid use in primary care: an overview and theory-based framework. Fam Med 2015;47:679-92.

40. Ivers N, Jamtvedt G, Flottorp S, et al. Audit and feedback: effects on professional practice and healthcare outcomes (Review). Cochrane Database Syst Rev 2012;6:CD000259.

41. Davis DA, Mazmanian PE, Fordis M, et al. Accuracy of physician self-assessment compared with observed measures of competence: a systematic review. JAMA 2006;296:1094-102.

42. Emanuel EJ, Ubel PA, Kessler JB, et al. Using behavioural economics to design physician incentives that deliver high-value care. Ann Intern Med 2016;164:114-9.

43. Arterburn $\mathrm{D}$, Wellman $\mathrm{R}$, Westbrook $\mathrm{E}$, et al. Introducing decision aids at Group Health was linked to sharply lower hip and knee surgery rates and costs. Health Aff 2012;31:2094-104.

44. Azjen I, Madden T. Prediction of goal-directed behaviour: attitudes, intentions and perceived behavioural control. ¿ Exp Soc Psychol 1986;22:453-74. 